\title{
Gonadal Development in Fish
}

\author{
Toshiya Nishimura Minoru Tanaka \\ Laboratory of Molecular Genetics for Reproduction, National Institute for Basic Biology, and Graduate School of \\ Advanced Study, SOKENDAI, Okazaki, Japan
}

\section{Key Words}

Gonad $\cdot$ Sex $\cdot$ Teleosts

\begin{abstract}
Vertebrate reproduction depends on the function of 2 distinct gametes, sperm and eggs, which develop in 2 different organs, the testis and the ovary. Testes and ovaries are composed of germ cells, supporting cells and interstitial cells. In this review, we describe the origin and the fate of these cell lineages and how they interact with each other to form sexually dimorphic reproductive organs in medaka. We delineate how the temporally different association and establishment of these lineages contribute to a variety of seemingly different sex differentiation processes among teleost fish. Thus, teleosts represent an intriguing group in which to study the fundamental processes of gonadal development through comparing conserved and unique mechanisms.
\end{abstract}

(c) 2014 S. Karger AG, Basel

The ovary and the testis are essential for gametogenesis. A mature ovary consists of an ovarian cavity, the germinal or surface epithelium, and the stromal compartment. In teleosts, germline stem cells and mitotically active oogonia reside in the germinal epithelium. This structure is equivalent to the surface epithelium in mammals. Follicles are present in the stromal compartment where oocytes grow and steroid hormones are produced. In the testis, spermatogenesis from germline stem cells to sperm occurs in tubules or lobules, and the interstitial tissue producing steroid hormone(s) resides between these structures. The 2 reproductive organs are grossly different, but they are both composed of developmentally common cell lineages, supporting cells, interstitial cells and germ cells.

In this review, we discuss the lineages of germ cells, supporting cells and interstitial cells and how they interact with each other to coordinate the sexually different organs in teleosts, especially focusing on studies in medaka, Oryzias latipes. Although the molecular mechanism(s) underlying gonadal development and sex differentiation in teleosts are still not as well understood as those in mammals, recent progress in transgenic technology and lineage analysis provides the opportunity to clarify these phenomena at the cellular level. In turn, these data will allow us to compare gonadal development in teleosts with that in other animals.

\section{Germ Cell Lineage}

Germ cells, critical for conveying the genetic information to the next generation, are very special in that they are segregated from other cells at a very early developmental stage when major positional information is being established, and migrate into the future gonadal area. Early-

\section{KARGER}

E-Mail karger@karger.com

www.karger.com/sxd
(C) 2014 S. Karger AG, Basel

1661-5425/14/0085-0252\$39.50/0
Minoru Tanaka

Laboratory of Molecular Genetics for Reproduction

National Institute for Basic Biology

5-1 Higashiyama Myodaiji-cho, Okazaki 444-8787 (Japan)

E-Mail mtanaka@ nibb.ac.jp 
stage germ cells that have not yet reached the gonad are called primordial germ cells (PGCs). PGCs in teleosts are morphologically identified and functionally specified by the allocation of cytoplasmic determinants, including the RNA-binding proteins NANOS, VASA and TUDOR which are localized on granule-like structures or nuage [Aoki et al., 2008]. This finding is similar to what has been observed in other lower vertebrates and Drosphila. In medaka, nanos 3 is the earliest marker so far examined for germ cells, and using this marker, PGCs can be first identified at an early gastrulation stage [Kurokawa et al., 2006]. The migration of PGCs consists of at least 3 mechanically distinct modes [Kurokawa et al., 2006; Herpin et al., 2008]. First, at an early gastrulation stage, PGCs actively migrate towards the marginal zone, a process which depends on the chemokine receptor CXCR4 and its ligand, SDF1A. Second, at the late gastrulation and early somitogenesis stages, PGC movement depends on the convergent movement of somatic cells. Third, after aligning bilaterally, PGCs, governed by interactions between CXCR 4 and SDF1B, resume active and directional migration towards the posterior end of the lateral plate mesoderm, where gonadal somatic precursors arise [Nakamura et al., 2006].

Germ cells begin to proliferate as a single gonadal primordium splits into 2 bilateral primordia. The germ cells in the primordia are characterized according to their types of division: type I and type II [Saito et al., 2007]. A type I division corresponds to a self-renewal division, resembling that of the typical stem cells. A type I germ cell divides into 2 daughter cells, each of which is readily surrounded by supporting cells. In type II division, germ cells synchronously and successively divide, are connected by intercellular bridges, and subsequently enter meiosis (fig. 1). An analysis of the zenzai mutant clearly shows that the germ cells undergoing type II divisions are the ones committed to gametogenesis [Saito et al., 2007].

In females, type II germ cells are first observed 2 days after the gonadal primordia form in the embryonic gonad. The commitment to type II division is related to the development of the ovarian structure. The female gonad produces oocytes with follicular cells (follicles) through type II division. By contrast, in males, germ cells keep dividing as type I germ cells during both the embryonic and the larval stages. Male germ cells do not begin to exhibit type II division until around 30-45 days post-hatching (dph), when the male-specific lobule, a unit of testicular structure in medaka, is formed (fig. 2) [Satoh and Egami, 1972; Satoh, 1974]. Nanos2, a good indicator of stem cells, is not detected in embryonic and larval germ cells. This

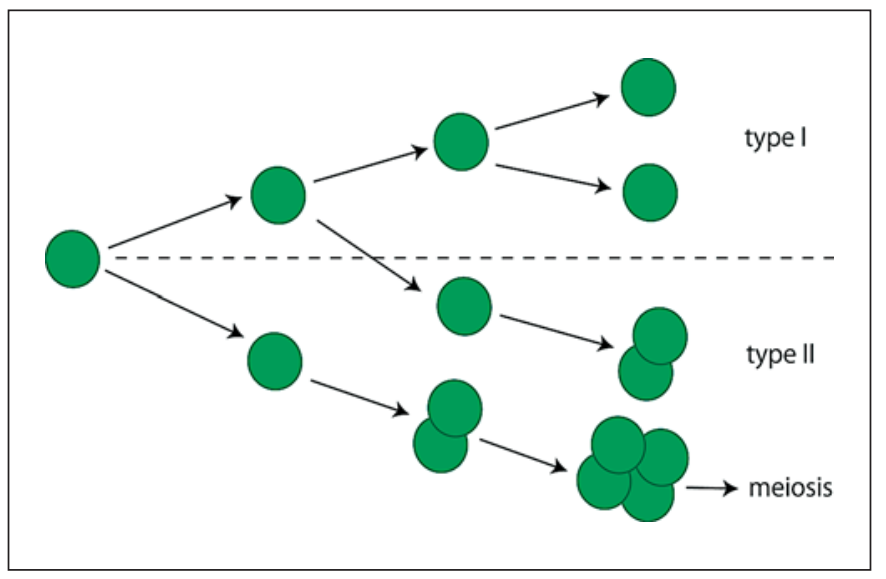

Fig. 1. Two types of division in embryonic medaka germ cells. Germ cells in embryonic gonads can be categorized according to their type of division. Type I is the stem cell-like division, in which a germ cell divides completely to generate 2 daughter cells. Type II is the gametogenesis-committed cystic division, in which germ cells divide synchronously and daughter cells are interconnected by intercellular bridges. Subsequently, type II germ cells enter meiosis.

may suggest that germ cells during the embryonic and larval stages are not fully developed as stem cells.

In the adult medaka testis, some populations of germ cells express nanos 2 and are preferentially located at the most distal ends of the lobules which are composed of sox 96 -expresssing cells (fig. 2). It is likely that the distal ends serve as niches for germline stem cells. The nanos2expressing germ cells correspond to what has been morphologically called type A spermatogonia and may have characteristics of the $A_{s}-A_{a l}$ spermatogonial populations in the mouse. However, unlike in the mouse, this cell population is not aligned by intercellular bridges. Type B spermatogonia divide as type II to produce mitotic germ cell cysts. After several rounds of type II division, they enter meiosis and differentiate into spermatocytes.

In the adult medaka ovary, early oogenesis occurs within a histological unit termed a germinal cradle. These units, like lobules in the testis, are composed of sox $9 \mathrm{~b}$-expressing cells (fig. 2). Within the ovarian germinal cradles, germ cells can be categorized into 3 groups [Nakamura et al., 2010, 2011]. The first is the single isolated germ cell (Gs type of germ cell) that expresses nanos 2 . This group contains germline stem cells as shown by clonal analysis, and is present in isolation surrounded by sox $9 b$-expressing supporting cells in the cradle. The second is the cyst-forming germ cell (Gcys) that is surrounded by sox $9 b$-expressing cells to form a cyst. The Gcys include mitotic germ cells and meiotic germ cells (from leptotene to pachytene stages). The third 


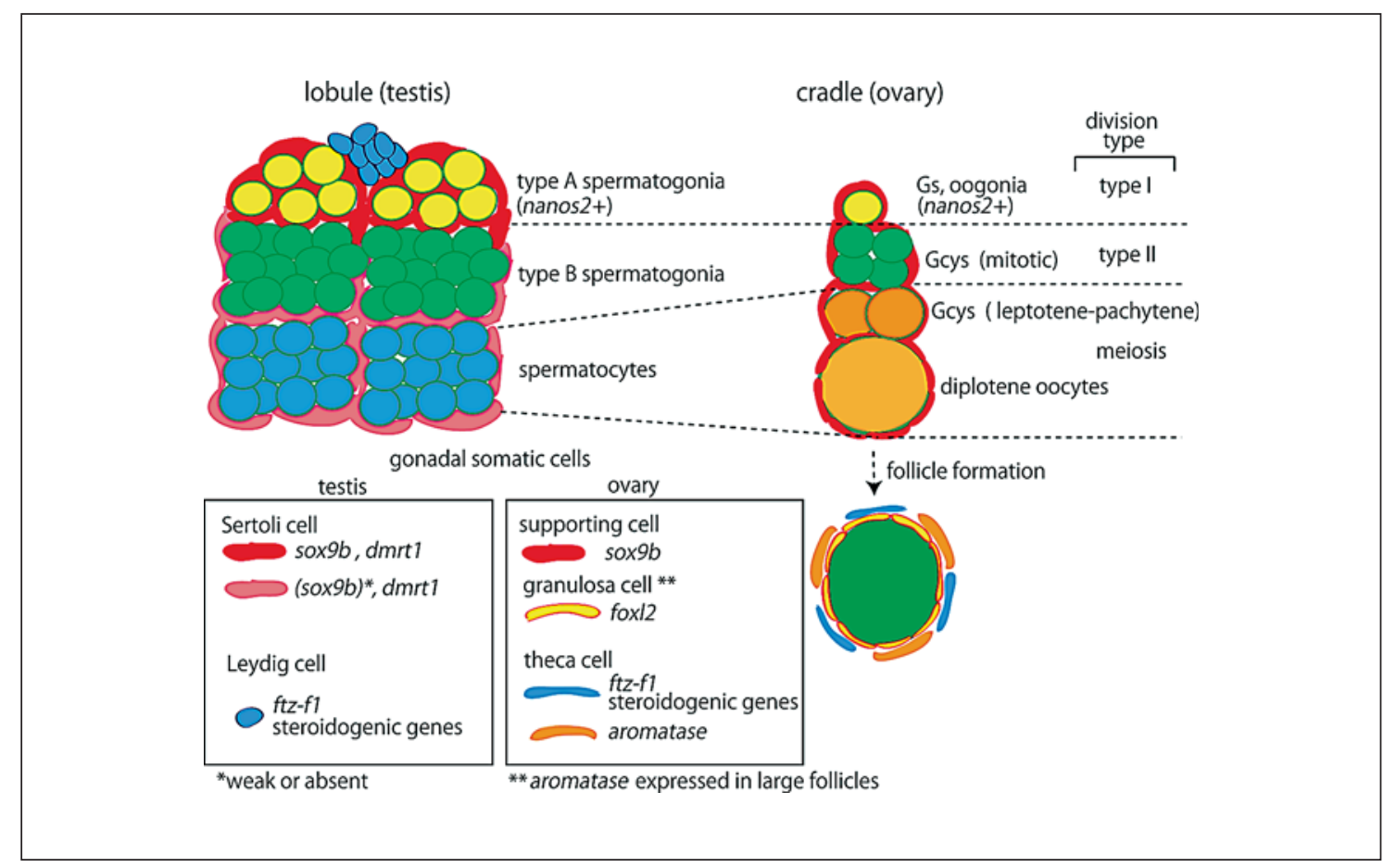

Fig. 2. Germ cells and somatic cells in the testis and ovary. Early spermatogenesis and oogenesis occur within structures called lobules and germinal cradles, which are composed of sox $9 b$-expressing supporting cells, in the testis and ovary, respectively. Type A spermatogonia and Gs or oogonia are single germ cells isolated by sox $9 b$-expressing cells. These germ cells express nanos 2 and undergo type I proliferation. Type B spermatogonia, spermatocytes and Gcys are cystic types of germ cells, and clusters of these germ cells are surrounded by sox $9 b$-expressing cells. Mitotic type B spermatogonia and Gcys are categorized as type II. Subsequently, type B spermatogonia enter meiosis and differentiate into spermatocytes. Gcys enter meiosis and differentiate into diplotene oocytes,

is the oocyte at the meiotic diplotene stage that is individually surrounded by sox $9 b$-expressing cells in the cradles. Subsequently, the diplotene oocytes exit from the cradles to form follicles in the stromal region (fig. 2).

The term 'germinal cradle' is conceptually different from the related term 'germinal nest'. The nest is histologically identified and is referred to as a cluster of mitotic germ cells, often accompanied by oocytes [Grier et al., 2007]. By contrast, the germinal cradle is the location of a niche containing a stem-type germ cell (Gs) that is surrounded by sox $9 b$-expressing cells [Nakamura et al., 2010]. Therefore, germinal cradles often have only a single Gs cell and do not form a 'nest'. Regarding division, type A spermatogonia and Gs are categorized as type I, and type B spermatogonia and mitotic Gcys are categorized as type II (fig. 2). Our results suggest that type A which are surrounded individually by sox $9 b$-expressing cells and exit from cradles to form follicles. In the testis, sox $9 b$ expression is higher in the Sertoli cells surrounding type A spermatogonia and becomes weaker or is absent as spermatogenesis proceeds within lobules. Leydig cells are located at interstitial regions within the testis and express $f t z-f 1$ and a set of steroidogenic genes necessary for the production of androgen. On the other hand, the expression levels of sox $9 b$ are uniform within cradles, and granulosa cells surrounding diplotene oocytes that have exited from the cradles downregulate sox $9 b$ expression and express foxl2. The forming follicles recruit at least 2 distinct types of theca cells - $f t z-f 1 /$ steroidogenic gene-expressing cells and aromatase-expressing cells.

spermatogonia include germline stem cells because some populations of type A spermatogonia strongly express nanos2. It is unknown, however, whether germ cells undergoing type II division retain stem cell characters or can dedifferentiate into germline stem cells, as reported in Drosophila [Brawley and Matunis, 2004; Kai and Spradling, 2004] and mice [Nakagawa et al., 2007].

\section{Lineage of Gonadal Somatic Cells in Vertebrates}

In amniotes, such as birds and mammals, the gonadal primordia originate as a pair of thickened rows of coelomic epithelial cells ventral to the mesonephros. Cells derived from the mesonephros tissue are important for testis development in both birds and mammals [Ayers et al., 
2013; Ungewitter and Yao, 2013]. The bipotential gonads of these vertebrates also show conserved dual structures, the medulla and cortex. It is generally said that testes develop from the medulla, and ovaries develop from the cortex [Merchant-Larios and Díaz-Hernández, 2013]. However, in teleosts, these 2 gonadal structures are not distinct. The gonadal primordia are observed as a cluster, extended along the anterior-posterior axis, composed of a few distinct cell types.

Some gonadal cell lineages come from the coelomic epithelium, and the mesonephros-derived cells contribute substantially to gonadal formation in mammals [Ungewitter and Yao, 2013]. However, there is no evidence for this pathway in teleosts where the gonadal primordium develops simultaneously with the formation of the coelomic epithelium and cavity. The pronephros and the interrenal organ, which are functionally similar to the mammalian kidney and adrenal gland, respectively, are located very anteriorly and form earlier than the gonadal primordium [Oguri, 1961; Nakamura et al., 2006]. From the pronephros, the nephric ducts extend posteriorly along the dorsal aorta [Ichimura et al., 2012] and reach the gonadal area concurrent with the onset of gonadal formation. In medaka, a few mesenchymal cells scatter around the nephric ducts (fig. 3) [Hamaguchi, 1982; Nakamura et al., 2006]. All of these developmental events and structures in medaka make the contribution of the pronephric/mesonephric cells to the gonad less obvious.

Recent advances in cell labeling and lineage tracing technologies enabled the detection of at least 2 different populations of gonadal somatic precursors in medaka embryos [Nakamura et al., 2006]. At stage (st.) 30, when PGCs are located on the lateral sides and move dorsally towards the prospective gonadal region along the developing hindgut, a population of gonadal precursor cells expressing $\mathrm{ftz}$ $f 1$, the medaka homolog of mammalian Ad4bp/Sf1, is observed adjacent to PGCs. The other population expresses $s o x 9 b$ and is located more dorsally, centering around the prospective gonadal area between the forming peritoneal wall and hindgut (fig. 3 ). These 2 populations meet PGCs and form the gonadal primordium by st.33 [Nakamura et al., 2006].

Interestingly, just when a single gonadal primordium forms at st. 33 , the analysis of $f t z-f 1$ and sox $9 b$ double-reporter transgenic embryos demonstrates transient activation of $f t z-f 1$ transcription in the sox $9 b$-expressing cells surrounding germ cells (fig. 4A-D). By the hatching stage, $f t z-f 1$ expression diminishes in the sox $9 b$-expressing cells as ftz-f1-expressing cells are positioned in the outer region of the gonad (fig. 4E). In mammals, the ex-

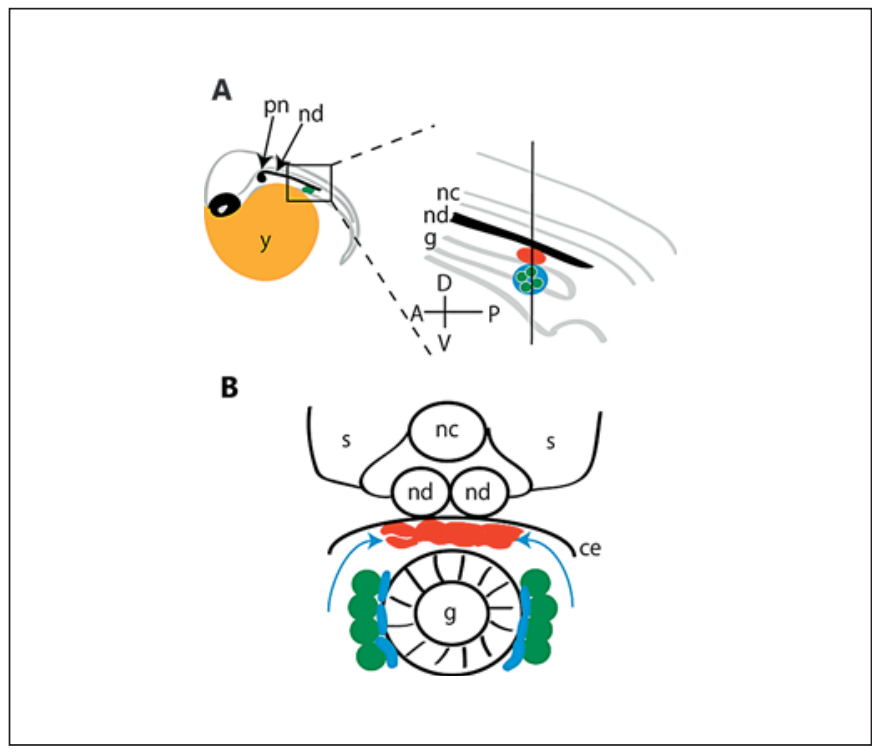

Fig. 3. Schematic representation of 2 distinct gonadal somatic precursors in medaka embryos. A A dorso-lateral view of medaka embryos at st.30. The boxed region is magnified on the right. Germ cells (green) and a population of gonadal somatic precursors expressing ftz-f1 (blue) are located near the forming hindgut (g). The other population expresses sox $9 b$ (red) and is located between the nephric duct (nd) and the forming hindgut. Note that the gonadal precursor cells are anatomically separate from the pronephros (pn). B A transverse section of an embryo at st.30. ftz-f1-expressing cells (blue) and germ cells (green) move to the prospective gonadal region, where the gonadal primordium is formed together with sox $9 b$-expressing cells (red). A = Anterior; ce = coelomic epithelium; $\mathrm{D}=$ dorsal; $\mathrm{nc}=$ notochord $\mathrm{P}=$ posterior; $\mathrm{s}=$ somite; $\mathrm{V}=$ ventral; $\mathrm{y}=$ yolk ball.

pression levels of $A d 4 b p / S f 1$, regulated by Pod1, determine the specification of both supporting cell and interstitial cell lineages [Cui et al., 2004]. Although sox9bexpressing cells eventually represent supporting cells at later stages and $f t z-f 1$ expression is a good indicator of interstitial cells [Kurokawa et al., 2007; Nakamura et al., 2010, 2011], regulation similar to mammals might function during the initial lineage-determination process at the very early stages of gonadal formation.

\section{Sertoli and Granulosa Cell Lineages in Teleosts}

In mammals, both Sertoli and granulosa cells share 2 common origins - mesenchymal cells of the genital ridge and coelomic epithelium [Karl and Capel, 1998; Mork et al., 2012]. However, the timing of migration from the coelomic epithelium and differentiation into 

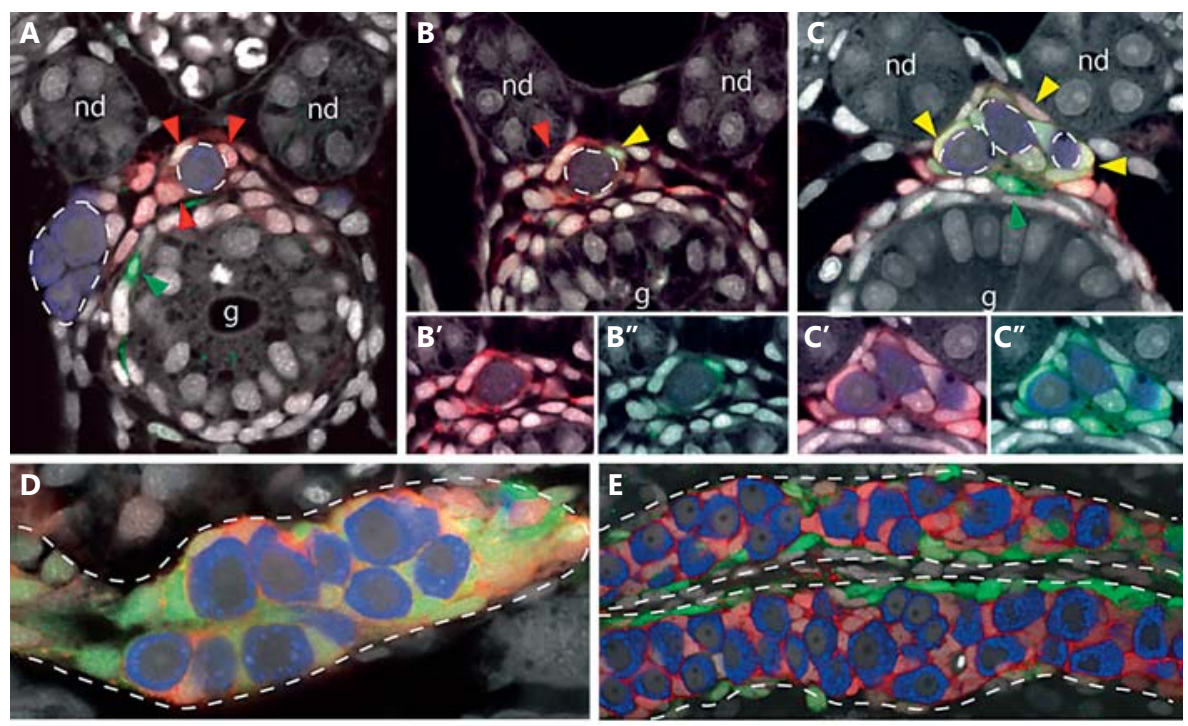

$\triangle$ sox9b-DsRed

$4 \mathrm{ftz}-\mathrm{f} 1-\mathrm{EGFP}$

4 sox9b-DsRed/ftz-f1-EGFP

Fig. 4. Lineage analysis of somatic cells using sox $9 b$-DsRed/ftz-f1EGFP in the forming medaka gonad. A-C Transverse sections of embryos at st.31-33. A At st.31, most germ cells (dashed lines) are still located at the lateral sides of the forming hindgut (g) and seem to be moving dorsally. A few germ cells begin to be surrounded by sox $9 b$-expressing cells (red arrowheads). At this stage, the supporting cells do not express $f t z-f 1$. The $f t z-f 1$-expressing cells (green arrowhead) are located around the forming hindgut as previously observed, but do not associate directly with germ cells. B-B" At st.32, sox $9 b$ and $f t z-f 1$ begin to be co-expressed in the supporting cell (yellow arrowhead), suggesting that sox $9 b$-expressing cells upregulate $f t z-f 1$ after they associate with germ cells. C-C' $\mathbf{C}^{\prime \prime}$ By st.33,

supporting cell lineages differs between the testis and ovary, suggesting that their origins are not equivalent [Ungewitter and Yao, 2013].

In teleosts, Sertoli and granulosa cells are suggested to share a common origin, namely, the supporting cells expressing the sox $9 b$ gene in the bipotential gonadal primordia. Interestingly, sox $9 b$ expression is found in both XX and XY supporting cells [Nakamura et al., 2008], an observation that is in sharp contrast to the situation in mammals, where sox9 is only expressed in Sertoli cells and is both required and sufficient for testicular development [Wagner et al., 1994; Vidal et al., 2001]. During testicular development, the lineage is straightforward. The sox $9 b$-expressing cells begin to ex- most sox $9 b$-expressing supporting cells express $f t z-f 1$ (yellow arrowheads). These observations indicate that germ cells are first surrounded by sox $9 b$-expressing cells located at a prospective gonadal region at st.30, followed by upregulation of $f t z-f 1$ by st.33. D, E Ventral view of the gonad (dashed lines) at st.33 (D) and the hatching stage (E). D Most supporting cells express both sox $9 \mathrm{~b}$ (red) and $f t z-f 1$ (green). E Around the hatching stage, while sox $9 b$ expressing cells (red) predominantly surround germ cells, ftz-f1expressing cells (green) are located relatively distal to sox $9 b$-expressing cells. Note that a single primordial gonad at st.33 (D) develops into bilateral lobes by the hatching stage (E). Blue: germ cells; gray: DAPI. nd = Nephric duct.

press $d m r t 1$, an indication of differentiation into Sertoli cells, and are actually located inside the lobules of the adult testis (fig. 2) [Nakamura et al., 2008, 2011].

As gonadal primordia develop into ovarian structures, the sox $9 b$-expressing cells constitute germline stem cell niches, or germinal cradles. As mentioned above, early oogenesis, from germline stem cells to early diplotene oocytes, proceeds in the cradles. Subsequently the diplotene oocytes and the surrounding somatic cells exit from the germinal cradle and recruit theca cells to form follicles. As a result, the follicles in the stromal compartment have 2 layers of somatic cells, outer theca cells and inner granulosa cells. During this step, the granulosa cells lose sox $9 b$ expression while 
foxl2, a marker of granulosa cells, is activated [Nakamoto et al., 2006; Herpin et al., 2013], suggesting granulosa cells originate from the sox $9 b$-expressing cells. Both follicular formation and oocyte exit from germinal cradles appears to depend upon a series of successive processes (fig. 2) [Nakamura et al., 2010, 2011]. Histological analysis of other teleost fish also supports this observation [Grier, 2000; Grier et al., 2007].

Interestingly, in the testis, sox $9 b$ expression is very intense in the Sertoli cells located most distally in the lobules. The distal region is predominately occupied by the most undifferentiated type of germ cells, type A spermatogonia [Nakamura et al., 2011]. In the ovary, sox $9 b$ is expressed in the germinal cradles representing niche regions (fig. 2). Collectively, these observations suggest that the common function of sox $9 b$-expressing cells may be the maintenance of stem-type germ cells during early gametogenesis. In support of this hypothesis, the heterozygous medaka sox $9 b$ mutant exhibits a gradual loss of germ cells [Nakamura et al., 2012b]. A similar phenotype is also reported in the conditional knockout mouse exhibiting disruption of both the Sox9 and Sox8 genes [Barrionuevo et al., 2009].

\section{Leydig and Theca Cell Lineages in Teleosts}

In teleosts, the knowledge of Leydig and theca cell lineages is still scarce, and the female steroidogenic cell populations seem to consist of several cell lineages, which makes the characterization complicated.

At 5-10 dph, steroidogenic genes necessary for the production of estradiol, e.g. $p 450 s c c / c y p 11 a 1, h s d 3 b$ and aromatase/cyp19a1, begin to be expressed on the ventral side of interstitial or epithelial cells in XX gonads [Suzuki et al., 2004; Nakamura et al., 2009; Nakamoto et al., 2010, 2012]. Subsequently, these cells differentiate into theca cells forming the outer layer of follicles (fig. 2). Unlike mammals, where estrogen production relies on granulosa cells in the inner follicular layer, the cells expressing aromatase at this early stage of medaka sex differentiation are the theca cells [Nakamura et al., 2009].

During testicular development, steroidogenic genes required for the production of steroid hormone(s), e.g. p450scc/cyp11a1 and $h s d 3 b$, begin to be expressed in presumptive Leydig cells located in the peripheral regions of the lobule structures from 15-20 dph [Nakamoto et al., $2010,2012]$. By $30-45$ dph, the number of Leydig cells increases and forms clusters in the interstitial space, a formation that is also observed in the adult testis [Satoh, 1974;
Kurokawa et al., 2007]. By this stage, the lobules have formed, and quiescent spermatogonia start to proliferate and initiate spermatogenesis (fig. 2) [Satoh and Egami, 1972; Satoh, 1974]. The rapid increase in the number of steroidogenic cells prior to spermatogenesis and lobule formation has also been reported in tilapia [Nakamura et al., 1998]. In mammals, Leydig cells develop in the interstitial space of the testis soon after the testicular cords are formed in the fetus [Morohashi et al., 2013]. Therefore, although the developmental timing is different, the formation of testicular structures seems temporally associated with the appearance of Leydig cells.

Steroidogenic genes are expressed in $f t z-f 1$-expressing cells during testicular development [Nakamoto et al., 2012]. Furthermore, in adult testes, $f t z-f 1$ and steroidogenic genes, such as $p 450 c 17 / c y p 17 a 1, h s d 11 b$ and $p 45011 b / c y p 11 b$, are expressed in Leydig cells at interstitial regions (fig. 2) [Kurokawa et al., 2007]. Together, these results suggest that ftz$f 1$ regulates a set of steroidogenic genes and that androgen production may occur in a single cell lineage of $f t z-f 1$ expressing cells. In rainbow trout, immunohistochemical analysis also revealed that P45011B/CYP11B, P450scc/ CYP11A1, HSD3B, and P450c17/CYP17A1 are all co-localized in interstitial Leydig cells [Kobayashi et al., 1998]. By contrast, during ovarian development, at least 2 types of theca cells seem to be present in medaka (fig. 2). One population of cells expresses $p 450 s c c$ and $h s d 3 b$ together with $f t z-f 1$, while the other population expresses only aromatase [Nakamoto et al., 2012]. Expression analysis using aromatase-reporter transgenic medaka has also revealed that p450c17 and aromatase are exclusively expressed [Nakamura et al., 2009]. These results suggest that theca cells may be derived from at least 2 distinct populations in medaka. Alternatively, the 2 types of theca cells may share a common precursor that expresses the $f t z-f 1$ gene, and that generates offspring capable of either maintaining or downregulating $f t z-f 1$ expression and initiating aromatase expression.

\section{Cell-Cell Interactions Directing Gonadal Formation}

During and after cell lineage specification, each cell lineage mutually interacts to coordinate gonadal sex differentiation and development. Histologically, germ cells are enclosed by sox $9 b$-expressing supporting cells in testis lobules and in ovarian follicles or sox $9 b$-expressing germinal cradles. The supporting cells reside close to interstitial cells. Next, we discuss the possible interactions among these different lineages during gonadal sex differentiation. 


\section{Germ Cell and Supporting Cell Interactions}

\section{Soma-to-Germ Cell Regulation}

The first distinct sign of gonadal sex differentiation is observed in germ cells. While germ cells in XX gonads initiate a type I to type II transition during the embryonic stage, germ cells in XY gonads keep dividing according to a type I division. As type II divisions rapidly increase the number of daughter cells through successive and synchronous divisions, females have more germ cells than males (fig. 1) [Saito et al., 2007]. Since medaka male sex determination is genetically triggered in supporting cells by the expression of $D M Y / d m r t 1 b Y$ on the Y chromosome [Matsuda et al., 2002; Nanda et al., 2002], it would be reasonable to propose that male supporting cells with $D M Y / d m r t 1 b Y$ expression regulate the transition from type I to type II division in germ cells.

One possible gene that mediates the regulation of germ cells by supporting cells is the gonadal-soma derived factor ( $g s d f$ ), which is highly expressed in XY supporting cells from 6 days post fertilization onwards and in Sertoli cells surrounding type A spermatogonia in adult testes [Shibata et al., 2010]. gsdf is a member of the transforming growth factor (TGF)- $\beta$ superfamily and is conserved among teleost species but not in tetrapods. $g s d f$ was first identified in rainbow trout as a gene expressed in the supporting cells surrounding PGCs, and could enhance the proliferation of PGCs and spermatogonia [Sawatari et al., 2007]. Functional analysis of $g s d f$ in vivo has not been performed in medaka, but based on the higher expression in XY supporting cells during gonadal sex differentiation and in Sertoli cells surrounding type A spermatogonia, $g s d f$ might suppress the transition from type I to type II division. However, this does not exclude the possibility that $g s d f$ regulates proliferation of a stem type and/or a cystic type of germ cells.

In the protogynous wrasse, $g s d f$ expression was upregulated during the sex reversal from female to male [Horiguchi et al., 2013]. Furthermore, it has been recently reported that $g s d f$ is a sex determination gene on the $\mathrm{Y}$ chromosome in a related species of medaka, Oryzias luzonensis [Myosho et al., 2012]. Considering that $g s d f$ is a secreted factor derived from supporting cells and is involved in germ cell proliferation, $g s d f$ could play a conserved role in male development across teleost fish, possibly via germ cell regulation.

Another soma-derived factor regulating the proliferation of germ cells is the anti-Müllerian hormone ( $\mathrm{amh}$ ). In mammals, AMH signaling is essential for the regres- sion of the primordial female reproductive tract, the Müllerian duct, in male development. However, in teleosts, a tissue equivalent to the Müllerian duct has not been identified. Studies of loss-of-function mutant medaka for AMH type II receptor (amhrII), named hotei, suggest that $\mathrm{AMH}$ signaling is necessary for the proper proliferation of germ cells [Morinaga et al., 2007; Nakamura et al., 2012a]. The hotei mutant displays hypertrophic gonads with an excess number of germ cells in both XX and XY fish. Both the AMH ligand and its receptor are expressed in the supporting cells, indicating that AMH activity indirectly affects germ cell regulation. One interesting observation from the hotei mutant is that germ cell regulation is related to sex differentiation. Approximately half of the XY mutants show male-to-female sex reversal mediated by germ cells (described below).

It has recently been reported that in Patagonian freshwater pejerrey (Patagonina hatcheri), a duplicated Ylinked anti-Müllerian hormone gene named $a m h y$, is expressed in somatic cells in a putative male gonad and has an important role in testicular development [Hattori et al., 2012]. Similar to medaka and other teleost fish, in pejerrey, male gonads expressing amhy also remain longer quiescent than female gonads, suggesting that a possible role of amhy is to restrict the germ cell proliferation [Strüssmann et al., 1997]. Furthermore, in tiger pufferfish, amhrII is allelic on the $\mathrm{X}$ and $\mathrm{Y}$ chromosomes, and the higher activity of amhrII corresponds to male development [Kamiya et al., 2012]. Therefore, the role of AMH signaling in the control of germ cell proliferation and/or testicular development may be conserved among teleost fish.

\section{Germ-to-Soma Cell Regulation}

Although germ cells are largely influenced by gonadal somatic cells, an analysis of germ cell-deficient medaka and hotei mutants indicates that germ cells also positively contribute to the gonadal sex differentiation in medaka [Kurokawa et al., 2007; Morinaga et al., 2007; Nakamura et al., 2012a]. The germ cell-deficient medaka develops as a male irrespective of genetic sex. The gonads show a tubule-like structure resembling a testis in terms of both structure and gene expression. This result indicates that germ cells are required for the formation of ovaries and the maintenance of granulosa and theca cells [Kurokawa et al., 2007]. The experiment using the hotei mutant also supports this notion. Maleto-female sex reversal in the XY hotei mutant is not observed in the absence of germ cells, suggesting that the ovarian formation in the sex reversal is mediated by 
germ cells, and is not a direct defect of the supporting cells [Morinaga et al., 2007; Nakamura et al., 2012a]. Interestingly, the supporting cells in the hotei sex-reversed ovary express both $d m r t 1$ and foxl2. Together, these data suggest that germ cells are necessary and sufficient for ovarian development. The importance of germ cells for the feminization of gonads is also reported in the zebrafish, Danio rerio [Slanchev et al., 2005; Siegfried and Nüsslein-Volhard, 2008; Dranow et al., 2013]. The establishment of dimorphic gonadal development requires proper crosstalk between germ cells and somatic cells in several teleost species [for review, see Tanaka et al., 2008; Saito and Tanaka, 2009].

The experiment of germ cell-ablation demonstrates another important point, the male predisposition of somatic cells. In medaka and zebrafish, gonadal somatic cells without germ cells develop into male cells, independent of sex chromosome constitution. However, recent studies using loach, Misgurnus angilicaudatus, and goldfish, Carassius auratus, show that germ cell-deficient gonads can differentiate into both ovary-like and testis-like gonads, suggesting that the male predisposition of somatic cells is somehow suppressed in these species [Fujimoto et al., 2010; Goto et al., 2012].

Do germ cells function differently in different teleost species? Interestingly, the timing of the formation of the ovarian cavity relative to germ cell development varies from species to species [Nakamura et al., 1998]. In medaka and zebrafish, meiotic entry and follicle formation precede ovarian cavity formation, a process which depends on estrogen [Suzuki et al., 2004]. In addition, the production of estrogen is dependent on germ cells because experiments indicate that germ cells are required for the maintenance of aromatase-expressing theca cells [Kurokawa et al., 2007; Nakamura et al., 2009]. By contrast, ovarian cavity formation occurs coincidentally with the appearance of meiotic germ cells in loach and goldfish, implying that estrogen secretion is independent of germ cells [Nakamura et al., 1998; Fujimoto et al., 2010]. Thus, it would be interesting to speculate that sufficient amounts of estrogen, produced by theca cells before the entry of meiosis in loach and goldfish, may induce early ovarian formation and suppress the autonomous differentiation of somatic cells towards the male phenotype. Further studies will be needed to explain the different roles of germ cells in the establishment of feminization. For example, it would be important to identify germ cell-derived secretion factors necessary for the maintenance of granulosa and theca cells.

Gonadal Development in Fish

\section{Supporting Cell and Interstitial Cell Interactions}

In the absence of germ cells, foxl 2 and aromatase are transiently expressed at the beginning of gonadogenesis [Kurokawa et al., 2007; Nakamura et al., 2009]. This observation indicates that the initiation of granulosa and theca cell development occurs without input from germ cells. Since in normal development, foxl2 and aromatase expression is not detected in XY gonads, this suggests that some interaction is present between supporting and interstitial cells. After the transient expression of foxl 2 and aromatase, foxl 2 expression is downregulated as $d m r t 1$ expression starts in the supporting cells. The dmrt1 expression also coincides with the decrease of aromatase expression in the interstitial cells [Kurokawa et al., 2007]. Although the factor(s) mediating these interactions have not yet been identified, the repression may be triggered by $d m r t 1$ function in the supporting cells. This hypothesis recalls a similar situation found in mouse, in which Dmrt 1 and Foxl2 mutually antagonize each other in order to balance the sexual phenotype of supporting cells [Uhlenhaut et al., 2009; Matson et al., 2011].

\section{Conclusion}

In this review, we have discussed the lineages of gonadal cells, including germ cells, supporting cells and interstitial cells, and have addressed the possible interactions coordinating these lineages during sex differentiation. In mammals, the process of gonadal sex differentiation can be divided into 2 steps: genetic regulation and endocrine regulation. Genetic regulation triggered by $S R Y$ initiates Sertoli and Leydig cell differentiation in the testis, and, in the absence of $S R Y$, induces granulosa and theca cell differentiation in the ovary. After each cell lineage has been established, endocrine regulation is required to maintain the sexual identity of each lineage [Morohashi et al., 2013].

The endocrine and genetic regulations also function in teleosts. However, these regulations contribute to the sex differentiation differently among teleost species. In meda$\mathrm{ka}$, the genetic regulation precedes the hormonal regulation. DMY/dmrt1bY acting in supporting cells regulates the male pathway and the transition of germ cells from type I to type II division, thus controlling sex differentiation of germ cells genetically. Without $D M Y / d m r t 1 b Y$, the interstitial cells initiate development to female interstitial cells and express aromatase. However, the establishment of female interstitial cells requires the feminized germ cells (meiotic germ cells with follicle cells). Loss of germ cells 
results in the failure to form ovaries. By contrast, in loach and goldfish, the initial genetic regulators in supporting cells may act more on the development of interstitial cells than germ cells. By the time meiotic germ cells appear, ovarian cavity formation has begun, suggesting that the endocrine system is established in loach and goldfish much earlier than in medaka. Thus, the hormonal regulation may contribute to ovarian formation more than in medaka, and this may explain why the gonad develops properly to either testis or ovary without germ cells.
Collectively, sex differentiation in a variety of teleost species stresses the equal importance of the 2 types of regulation. The identification of the molecules involved in the establishment of sexual identity through cell-cell interactions in different species will give us deep insights into understanding the conserved mechanisms underlying gonadal development and sex differentiation.

\section{References}

Aoki Y, Nagao I, Saito D, Ebe Y, Kinjo M, Tanaka M: Temporal and spatial localization of three germline-specific proteins in medaka. Dev Dyn 237:800-807 (2008)

- Ayers KL, Sinclair AH, Smith CA: The molecular genetics of ovarian differentiation in the avian model. Sex Dev 7:80-94 (2013).

- Barrionuevo F, Georg I, Scherthan H, Lécureuil C, Guillou F, et al: Testis cord differentiation after the sex determination stage is independent of Sox9 but fails in the combined absence of Sox9 and Sox8. Dev Biol 327:301-312 (2009).

- Brawley C, Matunis E: Regeneration of male germline stem cells by spermatogonial dedifferentiation in vivo. Science 304:1331-1334 (2004).

-Cui S, Ross A, Stallings N, Parker KL, Capel B, Quaggin SE: Disrupted gonadogenesis and male-to-female sex reversal in Pod 1 knockout mice. Development 131:4095-4105 (2004).

Dranow DB, Tucker RP, Draper BW: Germ cells are required to maintain a stable sexual phenotype in adult zebrafish. Dev Biol 376:43-50 (2013).

- Fujimoto T, Nishimura T, Goto-Kazeto R, Kawakami Y, Yamaha E, Arai K: Sexual dimorphism of gonadal structure and gene expression in germ cell-deficient loach, a teleost fish. Proc Natl Acad Sci USA 107:1721117216 (2010).

Goto R, Saito T, Takeda T, Fujimoto T, Takagi M, et al: Germ cells are not the primary factor for sexual fate determination in goldfish. Dev Biol 370:98-109 (2012).

Grier H: Ovarian germinal epithelium and folliculogenesis in the common snook, Centropomus undecimalis (Teleostei: Centropomidae). J Morphol 243:265-281 (2000).

Grier HJ, Uribe MC, Parenti LR: Germinal epithelium, folliculogenesis, and postovulatory follicles in ovaries of rainbow trout, Oncorhynchus mykiss (Walbaum, 1792) (Teleostei, protacanthopterygii, salmoniformes). J Morphol 268:293-310 (2007).

Hamaguchi S: A light- and electron-microscopic study on the migration of primordial germ cells in the teleost, Oryzias latipes. Cell Tissue Res 227:139-151 (1982).
Hattori RS, Murai Y, Oura M, Masuda S, Majhi SK, et al: A Y-linked anti-Müllerian hormone duplication takes over a critical role in sex determination. Proc Natl Acad Sci USA 109: 2955-2959 (2012).

-Herpin A, Fischer P, Liedtke D, Kluever N, Neuner C, et al: Sequential SDF1a and b-induced mobility guides medaka PGC migration. Dev Biol 320:319-327 (2008).

Herpin A, Adolfi MC, Nicol B, Hinzmann M, Schmidt C, et al: Divergent expression regulation of gonad development genes in medaka shows incomplete conservation of the downstream regulatory network of vertebrate sex determination. Mol Biol Evol 30:2328-2346 (2013).

Horiguchi R, Nozu R, Hirai T, Kobayashi Y, Nagahama Y, Nakamura M: Characterization of gonadal soma-derived factor expression during sex change in the protogynous wrasse, Halichoeres trimaculatus. Dev Dyn 242:388399 (2013).

Ichimura K, Bubenshchikova E, Powell R, Fukuyo Y, Nakamura T, et al: A comparative analysis of glomerulus development in the pronephros of medaka and zebrafish. PLoS One 7:e45286 (2012)

Kai T, Spradling A: Differentiating germ cells can revert into functional stem cells in Drosophila melanogaster ovaries. Nature 428:564-569 (2004).

Kamiya T, Kai W, Tasumi S, Oka A, Matsunaga $\mathrm{T}$, et al: A trans-species missense SNP in $a m h r 2$ is associated with sex determination in the tiger pufferfish, Takifugu rubripes (fugu). PLoS Genet 8:e1002798 (2012).

Karl J, Capel B: Sertoli cells of the mouse testis originate from the coelomic epithelium. Dev Biol 203:323-333 (1998).

Kobayashi T, Nakamura M, Kajiura-Kobayashi H, Young G, Nagahama Y: Immunolocalization of steroidogenic enzymes (P450scc, P450c17, P450arom, and 3B-HSD) in immature and mature testes of rainbow trout (Oncorhynchus mykiss). Cell Tissue Res 292:573-577 (1998).
Kurokawa H, Aoki Y, Nakamura S, Ebe Y, Kobayashi $\mathrm{D}$, Tanaka M: Time-lapse analysis reveals different modes of primordial germ cell migration in the medaka Oryzias latipes. Dev Growth Differ 48:209-221 (2006).

Kurokawa H, Saito D, Nakamura S, Katoh-Fukui Y, Ohta K, et al: Germ cells are essential for sexual dimorphism in the medaka gonad. Proc Natl Acad Sci USA 104:16958-16963 (2007)

Matson CK, Murphy MW, Sarver AL, Griswold MD, Bardwell VJ, Zarkower D: DMRT1 prevents female reprogramming in the postnatal mammalian testis. Nature 476:101-104 (2011).

Matsuda M, Nagahama Y, Shinomiya A, Sato T, Matsuda C, et al: $D M Y$ is a Y-specific DMdomain gene required for male development in the medaka fish. Nature 417:559-563 (2002).

Merchant-Larios H, Díaz-Hernández V: Environmental sex determination mechanisms in reptiles. Sex Dev 7:95-103 (2013).

- Morinaga C, Saito D, Nakamura S, Sasaki T, Asakawa S, et al: The hotei mutation of medaka in the anti-Müllerian hormone receptor causes the dysregulation of germ cell and sexual development. Proc Natl Acad Sci USA 104:9691-9696 (2007).

Mork L, Maatouk DM, McMahon JA, Guo JJ, Zhang $\mathrm{P}$, et al: Temporal differences in granulosa cell specification in the ovary reflect distinct follicle fates in mice. Biol Reprod 86:37 (2012).

Morohashi K, Baba T, Tanaka M: Steroid hormones and the development of reproductive organs. Sex Dev 7:61-79 (2013).

Myosho T, Otake H, Masuyama H, Matsuda M, Kuroki Y, et al: Tracing the emergence of a novel sex-determining gene in medaka, Oryzias luzonensis. Genetics 191:163-170 (2012).

Nakagawa T, Nabeshima Y, Yoshida S: Functional identification of the actual and potential stem cell compartments in mouse spermatogenesis. Dev Cell 12:195-206 (2007). 
Nakamoto M, Matsuda M, Wang DS, Nagahama Y, Shibata N: Molecular cloning and analysis of gonadal expression of Foxl2 in the medaka, Oryzias latipes. Biochem Biophys Res Commun 344:353-361 (2006).

- Nakamoto M, Fukasawa M, Orii S, Shimamori $\mathrm{K}$, Maeda T, et al: Cloning and expression of medaka cholesterol side chain cleavage cytochrome p450 during gonadal development. Dev Growth Differ 52:385-395 (2010).

- Nakamoto M, Fukasawa M, Tanaka S, Shimamori K, Suzuki A, et al: Expression of $3 \beta$-hydroxysteroid dehydrogenase ( $h s d 3 b$ ), star and ad $4 \mathrm{bp} / \mathrm{sf}-1$ during gonadal development in medaka (Oryzias latipes). Gen Comp Endocrinol 176:222-230 (2012).

- Nakamura M, Kobayashi T, Chang XT, Nagahama Y: Gonadal sex differentiation in teleost fish. J Exp Zool 281:362-372 (1998).

- Nakamura S, Kobayashi D, Aoki Y, Yokoi H, Ebe $\mathrm{Y}$, et al: Identification and lineage tracing of two populations of somatic gonadal precursors in medaka embryos. Dev Biol 295:678688 (2006)

- Nakamura S, Aoki Y, Saito D, Kuroki Y, Fujiyama A, et al: Sox9b/sox9a2-EGFP transgenic medaka reveals the morphological reorganization of the gonads and a common precursor of both the female and male supporting cells. Mol Reprod Dev 75:472-476 (2008).

- Nakamura S, Kurokawa H, Asakawa S, Shimizu $\mathrm{N}$, Tanaka M: Two distinct types of theca cells in the medaka gonad: germ cell-dependent maintenance of cyp19a1-expressing theca cells. Dev Dyn 238:2652-2657 (2009).

- Nakamura S, Kobayashi K, Nishimura T, Higashijima S, Tanaka M: Identification of germline stem cells in the ovary of the teleost medaka. Science 328:1561-1563 (2010).

- Nakamura S, Kobayashi K, Nishimura T, Tanaka $\mathrm{M}$ : Ovarian germline stem cells in the teleost fish, medaka (Oryzias latipes). Int J Biol Sci 7: 403-409 (2011).
- Nakamura S, Watakabe I, Nishimura T, Picard JY, Toyoda A, et al: Hyperproliferation of mitotically active germ cells due to defective antiMüllerian hormone signaling mediates sex reversal in medaka. Development 139:22832287 (2012a).

Nakamura S, Watakabe I, Nishimura T, Toyoda A, Taniguchi Y, Tanaka M: Analysis of medaka sox9 orthologue reveals a conserved role in germ cell maintenance. PLoS One 7:e29982 (2012b).

Nanda I, Kondo M, Hornung U, Asakawa S, Winkler C, et al: A duplicated copy of $d m r t 1$ in the sex-determining region of the $\mathrm{Y}$ chromosome of the medaka, Oryzias latipes. Proc Natl Acad Sci USA 99:11778-11783 (2002).

Oguri M: Histomorphology on the kidney and adrenal gland of medaka, Oryzias latipes. Bull Jpn Soc Sci Fish 27:1058-1062 (1961).

-Saito D, Tanaka M: Comparative aspects of gonadal sex differentiation in medaka: a conserved role of developing oocytes in sexual canalization. Sex Dev 3:99-107 (2009).

Saito D, Morinaga C, Aoki Y, Nakamura S, Mitani $\mathrm{H}$, et al: Proliferation of germ cells during gonadal sex differentiation in medaka: insights from germ cell-depleted mutant zenzai. Dev Biol 310:280-290 (2007).

Satoh N: An ultrastructural study of sex differentiation in the teleost Oryzias latipes. J Embryol Exp Morphol 32:195-215 (1974).

-Satoh N, Egami N: Sex differentiation of germ cells in the teleost, Oryzias latipes, during normal embryonic development. J Embryol Exp Morphol 28:385-395 (1972).

- Sawatari E, Shikina S, Takeuchi T, Yoshizaki G: A novel transforming growth factor- $\beta$ superfamily member expressed in gonadal somatic cells enhances primordial germ cell and spermatogonial proliferation in rainbow trout (Oncorhynchus mykiss). Dev Biol 301:266275 (2007).
Shibata Y, Paul-Prasanth B, Suzuki A, Usami T, Nakamoto $M$, et al: Expression of gonadal soma derived factor (GSDF) is spatially and temporally correlated with early testicular differentiation in medaka. Gene Expr Patterns 10:283-289 (2010)

Siegfried KR, Nüsslein-Volhard C: Germ line control of female sex determination in zebrafish. Dev Biol 324:277-287 (2008).

Slanchev K, Stebler J, de la Cueva-Méndez G, Raz E: Development without germ cells: the role of the germ line in zebrafish sex differentiation. Proc Natl Acad Sci USA 102:4074-4079 (2005).

Strüssmann CA, Saito T, Usui M, Yamada H, Takashima F: Thermal thresholds and critical period of thermolabile sex determination in two atherinid fishes, Odontesthes bonariensis and Patagonina hatcheri. J Exp Zool 278:167177 (1997)

-Suzuki A, Tanaka M, Shibata N: Expression of aromatase mRNA and effects of aromatase inhibitor during ovarian development in the medaka, Oryzias latipes. J Exp Zool Comp Exp Biol 301A:266-273 (2004).

Tanaka M, Saito D, Morinaga C, Kurokawa H: Cross talk between germ cells and gonadal somatic cells is critical for sex differentiation of the gonads in the teleost fish, medaka (Oryzias latipes). Dev Growth Differ 50:273-278 (2008).

Uhlenhaut NH, Jakob S, Anlag K, Eisenberger T, Sekido R, et al: Somatic sex reprogramming of adult ovaries to testes by FOXL2 ablation. Cell 139:1130-1142 (2009).

Ungewitter EK, Yao HHC: How to make a gonad: cellular mechanisms governing formation of the testes and ovaries. Sex Dev 7:7-20 (2013).

-Vidal VP, Chaboissier MC, de Rooij DG, Schedl A: Sox9 induces testis development in XX transgenic mice. Nat Genet 28:216-217 (2001).

-Wagner T, Wirth J, Meyer J, Zabel B, Held M, et al: Autosomal sex reversal and campomelic dysplasia are caused by mutations in and around the SRY-related gene SOX9. Cell 79: 1111-1120 (1994). 